\title{
"Il ridervi de la goffezza del dire": Niccolò Franco et la satire napolitaine du pétrarquisme
}

\author{
ROLAND BÉHAR \\ École Normale Supérieure, Paris
}

This essay explores the Neapolitan background of Niccolo Franco and argues that although the main purpose of his Il Petrarchista (Venice, 1539) was certainly a kind of Erasmian and Aretinian satire of the Petrarchist mode which grounded Pietro Bembo's Prose della volgar lingua (1525), still not enough critical attention has been given to the Neapolitan background of Francos education (until 1536), which is essential to an understanding of Il Petrarchista. Indeed, not only did Franco praise the Neapolitan heirs of Pontano's and Sannazaro's Academy (Epicuro, Rota and Tansillo), contrasting them with the Venetian imitation of Bembo's canon, but he himself was also imbued with that Neapolitan humanism which had used poetical satire to shape the intellectual space in sixteenthcentury Italy. This paper recalls some passages of Fabrizio Luna's Vocabulario (1536) and, especially, of Benedetto Di Falco's Rimario (1535), in order to compare them with Franco's literary positionswhose conception of imitatio derived also from Erasmus'. In conclusion, this paper suggests that the satirical scheme of Di Falco's Rimario formulated in nuce a cultural stance that Niccolo Franco would later reinforce in his Il Petrarchista.

L'article explore le contexte napolitain de l'œuvre de Niccolò Franco et montre que la formation que Franco reçut à Naples, jusqu'en 1536, est essentielle à la compréhension de son ouvrage, même s'il ne fait aucun doute qu' Il Petrarchista (Venise, 1539) fait, dans la lignée d'Érasme et de l'Arétin, la satire de la mode pétrarquiste lancée par les Prose della volgar lingua (1525) de Pietro Bembo. En effet, Franco ne fait pas seulement l'éloge des héritiers napolitains de l'académie de Pontano et de Sannazar (Epicuro, Rota et Tansillo), en les opposant au canon vénitien de l'imitation de Bembo. Lui-même est imprégné de la leçon de l'humanisme napolitain, qui a eu recours à la satire poétique pour défendre sa place dans le champ intellectuel de l'Italie du Cinquecento. L'article rappelle quelques passages $d u$ Vocabulario (1536) de Fabrizio Luna et, surtout, du Rimario (1535) de Benedetto Di Falco, pour les comparer aux positions littéraires de Franco - dont la théorie de l'imitatio a été par ailleurs influencée par Érasme. Finalement, on propose que le schéma satirique formulé par Di Falco dans le Rimario contient in nuce une position que Niccolò Franco développa et renforça ensuite dans Il Petrarchista.

e pétrarquisme fut à la fois une " manière littéraire » et un " langage
mondain "1. Il en va du terme comme de celui d'« humanisme ", qui l'a

1. Cf. Ferdinando Neri, "Le rime ultime di Gaspara Stampa ", in Saggi di letteratura italiana francese inglese (Naples : Loffredo, 1936), 271. 
peut-être inspiré ${ }^{2}$. Si la notion, ainsi que celle, contraire et plus controversée, $\mathrm{d}^{\prime}$ " antipétrarquisme », fut formée au XIX ${ }^{\mathrm{e}}$ siècle $^{3}$, le personnage social et littéraire du "pétrarquiste » exista dès le moment où le magistère de Bembo encouragea poètes érudits et courtisans à en adopter les normes ${ }^{4}$. On put dès lors s'en prendre aux imitateurs sans critiquer le modèle. Dès les premières parodies, la séparation fut relativement nette. La critique ne visait pas l'inspiration, qui avait été celle de Pétrarque ou de Bembo, mais le manque d'inspiration de ceux qui pillaient leurs vers pour en orner leurs propres discours dans l'espoir d'atteindre ce que Giovanni Della Casa, dans son Galateo (1558), nommerait l'arte di essere grazioso.

Depuis 1501, année de la publication du Pétrarque avec lequel bien des courtisans se faisaient représenter le tenant à la main ${ }^{5}$, Bembo formulait les principes du «pétrarquisme " dans les ouvrages qu'il publiait chez Alde, à Venise. Le rapide succès de la mode du pétrarquisme dans la société incita à le critiquer et à le parodier. Une génération après le Pétrarque publié par Alde en format in-octavo de poche (tascabile), après les Asolani (1505) et les Prose della volgar lingua (1525), le " pétrarquisme ", compris comme imitation servile du poète de Laure, devint l'objet de railleries, voire de satires, notamment sous la plume de l'Arétin ${ }^{6}$. Ce dernier ne s'opposait pas à Bembo ; il en fut

2. Cf. Augusto Campana, "The Origin of the Word "Humanist" ", Journal of the Warburg and Courtauld Institutes 9 (1946) : 60-73 et Giuseppe Billanovich, "Auctorista, humanista, orator », Rivista di cultura classica e medioevale 7 (1965) : 155-160, rééd. in Giuseppe Billanovich, Itinera. Vicende di libri e di testi, éd. Mariarosa Cortesi, 2 vol. (Rome : Edizioni di Storia e Letteratura, 2004), 2 : 187-208.

3. Arturo Graf, Petrarchismo ed antipetrarchismo nel Cinquecento (Rome : Tip. della Camera dei Deputati, 1886).

4. Stefano Jossa, «Bembo and Italian Petrarchism », in The Cambridge Companion to Petrarch, éd. Albert Russell Ascoli et Unn Falkeid (New York : Cambridge University Press, 2015), 191-200.

5. Les portraits col Petrarchino sont nombreux : Parmigianino, Licinio, Puligo, Del Sarto ou Bronzino en peignent dès les années 1520 - au moment même de la publication des Prose —, et Bronzino en perpétue la mode jusque dans les années 1550, avec son portrait de la poétesse Laura Battiferri. Dès 1530, Jean Clouet en diffuse aussi la mode à l'étranger, dans un portrait, aujourd'hui conservé à Windsor Castle, d'un jeune homme tenant à la main un livre arborant le titre PETRARCA. Voir Arnaldo Di Benedetto, «Un'introduzione al petrarchismo cinquecentesco », Italica 83.2 (2006) : 170-215, ici 178-179.

6. On peut en particulier se souvenir de telle lettre de l'Arétin au duc de Mantoue (XXIV, 2 juin 1531), Pietro Aretino, Lettere. Libro I, éd. Paolo Procaccioli [Rome : Salerno, 1997], I : 78 : « Fu pure strano umore il mio, in non aver voluto usare il sermon de la patria: e ciò è stato per le notomie che ogni pedante fa su la favella Toscana. Se l'anima del Petrarca e del Boccaccio, nel mondo suo, è tormentata, come son le loro 
même le complice, semble-t-il, notamment pour disqualifier son adversaire Antonio Brocardo, tenu par Bernardo Tasso et Sperone Speroni pour un poète prometteur. Brocardo mourut en 1531. S’il avait vécu plus longtemps, il aurait pu développer une poésie lyrique inspirée des Anciens, donc libérée d'une imitation rigoureuse du pétrarquisme ${ }^{7}$.

opere nel nostro, debbono rinnegare il battesimo. » ( "J'ai été pris d’une étrange humeur, lorsque je n’ai pas voulu employer la langue de la patrie, et cela vient du fait des anatomies que tout pédant fait maintenant de son parler toscan. Si l'âme de Pétrarque et celle de Boccace sont tourmentées dans leur monde, comme leur œuvres le sont dans le nôtre, ils doivent renier leur baptême. » [sauf indication contraire, les traductions sont les miennes]), ou telle autre encore, à Lodovico Dolce (CLV, 25 juin 1537), ou encore celle-ci, adressée précisément à Niccolò Franco, Aretino, Lettere : I : 229-232 : « Così doveria fare chi si vale di quel poeta e di questo; $e$, col torgli solamente i fiati degli spiriti, uscir fuora con una armonia formata da le voci degli organi propri. Perchè le orecchie altrui sono oggimai sazie degli "uopi" e degli "altresi", e il vedergli per i libri movono a riso ne la maniera che moveria un cavaliere, comparendo in piazza in giornea tutta tempestata di tremolanti d'oro e con la berreta a tagliere, onde si credebbe che egli fosse impazzito o mascarato. [...] E per dirvelo, il Petrarca e il Boccaccio sono imitati da chi esprime i concetti suoi con la dolcezza e con la leggiadria con cui dolcemente e leggiadramente essi andarono esprimendo $i$ loro, $e$ non da chi gli saccheggia, non pur dei "quinci" e dei "quindi" e dei "soventi" e degli "snelli", ma dei versi interi. E quando sia che il diavolo ci aciechi a trafugare qualcuno, sforziamoci di somigliarci a Vergilio, che svaligiò Omero, e al Sanazaro, che l'accoccò a Vergilio, onde hanno avanzato de l'usura; e saracci perdonato. Ma il cacar il sangue dei pedanti che vogliono poetare, rimoreggia de l'imitazione, e mentre ne schiamazzano negli scartabelli, la trasfigurano in locuzione, ricamandola con parole tisiche in regola" (" Ainsi devrait faire qui se réclame de ce poète et de cette manière. Rien qu'en prenant les souffles des esprits, il devrait exhaler une harmonie composée par les voix de ses propres organes. En effet, les oreilles d'autrui sont déjà rassasiées par les "uopi" et les "altresì", et quand on les voit dans les livres, ils suscitent le rire comme le suscite un chevalier apparaissant sur la place dans un manteau tout incrusté d'oripeaux d'or, avec un bonnet plat, ce qui ferait qu'on le tiendrait pour fou ou masqué. [...] Pour vous le dire autrement : Pétrarque et Boccace sont imités par qui exprime ses idées avec la douceur et avec la grâce qui furent les leurs, et non par qui met à sac non seulement les "quinci" et les "quindi", les "soventi" et les "snelli", mais aussi des vers entiers. Ainsi, lorsque le diable nous aveugle et nous convertit en voleurs, efforçons-nous de ressembler à Virgile, qui dévalisa Homère, et à Sannazar, qui s'est attaché à Virgile, dont ils ont exagéré l'usure, et il nous sera pardonné. Mais chier le sang comme les pédants qui se prétendent poètes fait seulement tomber en discrédit l'imitation et tandis qu'ils s'en réclament dans leurs manuels, ils la transfigurent en une locution et l'enrichissent des mots arides de leurs lois »). Sur l'Arétin anti-pétrarquiste, voir, dans le présent numéro de Renaissance and Reformation / Renaissance et Réformation, l'article de Marco Faini, " "E poi in Roma ognuno è Aretino": Pasquino, Aretino and the Concealed Self».

7. Voir Giovanni Ferroni, Dulces lusus. Lirica pastorale e libri di poesia nel Cinquecento (Alessandria : Edizioni dell’Orso, 2012), 43-52. 
Un jeune écrivain de Bénévent, Niccolò Franco (1515-1570) ${ }^{8}$, qui résida à Naples de 1531 à 1536 (où son frère latiniste Vincenzo l'introduisit dans les cercles pontaniens), composa l'une des plus importantes parodies du pétrarquisme de la première moitié du XVI siècle : Il Petrarchista (Venise, 1539), par laquelle il donna un nom à la mode. Il renouait avec la veine de l'Arétin - dont il fut le secrétaire à Venise avant de devenir son pire ennemi. Cette parodie ayant déjà été étudiée ${ }^{9}$, on s'attachera ici au rôle qu’a eu dans sa genèse le développement antérieur, à Naples, de la critique du pétrarquisme. Non content de parodier les commentaires de Pétrarque - parmi lesquels figurait, outre ceux d'Alessandro Vellutello (Il Petrarcha, 1525) et de Sebastiano Fausto da Longiano (Il Petrarcha, 1532), celui, napolitain, de Giovanni Andrea Gesualdo (Il Petrarcha, 1533) ${ }^{10}$ - Franco développa une satire qui, si elle plongeait ses racines dans les cercles septentrionaux proches de Bembo, s'inspirait aussi d'éléments trouvés à Naples.

\section{Niccolò Franco et les modèles d'Il Petrarchista}

Muni d'une lettre de recommandation à l'attention du Titien, Franco arriva à Venise en 1536. S'engageant dans le dur "mestiere di scrivere ", il se mit à publier : d'abord, Il Tempio di Amore, en 1536, plagiat d'une œuvre de même

8. Voir, pour sa biographie, Carlo Simiani, La vita e le opere di Nicolò Franco (Turin-Rome : L. Roux et Cie., 1894) et Paul F. Grendler, Critics of the Italian World (1530-1560) (Madison : University of Wisconsin Press, 1969).

9. Voir en particulier Roberto L. Bruni, « Parodia e plagio nel Petrarchista di Nicolò Franco », Studi e problemi di critica testuale (Bologna) 20 (1980) : 61-83. La parodie commence par la reprise, sur la page de titre, d'un portrait de Pétrarque de profil très similaire à celui employé pour l'édition du commentaire de Vellutello publié à Venise chez Giolito, en 1538. De même, dans le texte, Franco reprenait textuellement des passages des Prose de Bembo ou de Gesualdo ; cf. Niccolò Franco, Il Petrarchista, éd. Roberto L. Bruni (Exeter : University of Exeter, 1979), 19 et 22-24. Voir Pasquale Sabbatino, «Il Petrarchista di Nicolò Franco ", in Petrarca e Napoli, Atti del convegno, Napoli, 8-11 dicembre 2004, éd. Michele Cataudella (Pise-Rome : Fabrizio Serra, 2006).

10. Voir Gino Belloni, Laura tra Petrarca e Bembo (Padoue : Antenore, 1992), Catharina Busjan, Petrarca-Hermeneutik: Die Kommentare von Alessandro Vellutello und Giovan Andrea Gesualdo im epochalen Kontext (Berlin-Boston : Walter De Gruyter, 2013), et Roberto L. Bruni, « Parodia e plagio nel Petrarchista di Nicolò Franco ». 
"Il ridervi de la goffezza del dire" : Niccolò Franco et la satire napolitaine du pétrarquisme 191

titre du Napolitain Iacopo Campanile ${ }^{11}$; puis, en avril 1539, Le pistole vulgari et, en septembre de la même année, les Dialoghi piacevoli, où il dressa un Parnasse poétique dont il attribua la première place à Tansillo ; enfin, en octobre de la même année, Il Petrarchista, récit du pèlerinage de deux admirateurs fétichistes de Pétrarque se rendant en Avignon, sur les lieux de la passion amoureuse du poète de Laure ${ }^{12}$.

Présentes dans Le pistole vulgari et les Dialoghi de Franco ${ }^{13}$, les traces d'érasmisme sont sensibles dans la genèse d'Il Petrarchista. Dans une brève missive publiée à la fin de ce dialogue, Franco s'adresse à son ami Francesco Alunno ${ }^{14}$ pour mieux expliquer son ouvrage : ce «Dialogo », distinct des autres dialogues publiés ensemble la même année, doit en être rapproché ${ }^{15}$, de même

11. Voir Carlo Simiani, "Un plagio di Niccolò Franco ", Rassegna critica della letteratura italiana 5 (1900) : 19-26, et Alessandro Capata, « Nicolò Franco e il plagio del Tempio d'amore », in Furto e plagio nella letteratura del Classicismo, éd. Roberto Gigliucci (Rome : Bulzoni, 1998), 219-232.

12. Voir Joseph Burney Trapp, "Petrarchan Places. An Essay in the Iconography of Commemoration ", Journal of the Warburg and Courtauld Institutes 69 (2006) : 1-50, Pasquale Sabbatino, « In pellegrinaggio alle dimore poetiche del Petrarca. Gli itinerari, le reliquie di Laura e il ritratto di Simone Martini nel "Petrarchista” di Nicolò Franco », Studi rinascimentali 1 (2003) : 61-82, Rinaldo Rinaldi, « "O petrarchisti, che vi venga il cancaro". Niccolò Franco e la parte del vero nel codice lirico cinquecentesco ", in $I l$ Petrarchismo : un modello di poesia per l'Europa, éd. Loredana Chines (Rome : Bulzoni, 2006), 443-464 et Harald Hendrix, "The Early Modern Invention of Literary Tourism: Petrarch's Houses in France and Italy ", in Writers' Houses and the Making of Memory, éd. Harald Hendrix (New York : Routledge, 2007), 15-29.

13. Voir Silvana Seidel Menchi, Erasmo in Italia. 1520-1580 (Turin : Bollati-Boringhieri, 1987) et Ugo Rozzo, "Erasmo espurgato dai Dialoghi piacevoli di Nicolò Franco », in Erasmo, Venezia e la cultura padana nel '500, éd. Achille Olivieri (Rovigo : Minelliana, 1995), 191-208. L'influence du modèle érasmien est sujette à débat. Cairns a suggéré que c'est à Franco qu'il conviendrait d'attribuer l'idée du recueil de lettres vernaculaires (libro di lettere) comme formule éditoriale, inspirée par le De conscribendis epistolis d'Érasme, et non à l'Arétin, dont il était le secrétaire au moment de la parution du Primo libro de le lettere (1538). Voir Christopher Cairns, « Niccolò Franco, l'umanesimo meridionale e la nascita dell'epistolario in volgare ", in Cultura meridionale e letteratura italiana. I modelli narrativi dell'età moderna, éd. Pompeo Giannantonio (Naples : Loffredo, 1985), 119-128, ainsi que Paolo Procaccioli, «Cosí lavoravano per Aretino. Franco, Dolce e la correzione di Lettere », Filologia e Critica 21 (1996) : 264-280.

14. Cette même année, Alunno avait publié ses Osservationi sopra il Petrarca (Venise, 1539), vaste entreprise d'analyse systématique des vers de Pétrarque.

15. Voir Niccolò Franco, Il Petrarchista, Dialogo di. M. Nicolo Franco, Nel quale si scuoprono nuoui Secreti sopra il Petrarca. E si dãno a leggere molte lettere, che il mede[si]mo Petrarca, in lingua Thoscana scrisse a diuerse persone. Cose rare, ne mai piu date a luce [...] (Venetiis : apud Ioannem Giolitum de Ferrariis, 
que le Ciceronianus s'apparente aux autres dialogues satiriques d'Érasme. Sous la prose du Petrarchista perce le souvenir du dialogue érasmien qui, en 1528, à peine une dizaine d'années plus tôt, avait caractérisé le fétichiste cicéronien, sous les traits de Nosoponus. Or, ce personnage ne faisait quappliquer la doctrine de l'imitation de Cicéron que Bembo avait théorisée dans son épître De imitatione (1512) adressée à Jean-François Pic de La Mirandole — réplique aux critiques qu'Ange Politien, en une épître à Paolo Cortese, avait élevées contre l'imitation exclusive de Cicéron. Cornelio Castaldi, mort en 1537, avait déjà critiqué les "imitatori del Petrarca " en un capitolo reprenant les termes par lesquels Politien avait stigmatisé les imitateurs de Cicéron, "similes vel psittaco vel picae ${ }^{16}$. De même, le dialogue parodique sur le " pétrarquisme » de Franco dessinait les contours d'une nouvelle manière d'être, d'un nouvel ethos, qui était comme le pendant vernaculaire du Ciceronianus d'Érasme - et ce d'autant plus que l'« inventeur » théorique de l'imitation de Cicéron et de celle de Pétrarque était le même Bembo ${ }^{17}$.

Le projet du livre de Franco était à l'origine double, écrivait-il à la fin de son épître liminaire ${ }^{18}$. Son propos était de tourner en dérision la goffezza, la

1539), fol. $54 v^{\circ}$. Nous citons d'après l'editio princeps. L'ouvrage fut réimprimé, toujours chez Giolito de Ferrari, en 1541 et en 1543. Une édition curieuse est celle de Venise: appresso Barezzo Barezzi, 1623 : Li due Petrarchisti, dialoghi di Nicolò Franco, et di Ercole Giovannini. Ne' quali con vaga dispositione si scuoprono bellissime fantasie, nuovi, et ingegnosi secretti sopra il Petrarca e si danno a leggere molte lettere missive e responsive, che lo stesso Petrarca in lingua Toscana scrisse al Re Roberto di Napoli, a D. Gherardo Petrarca, a Benvenuto da Imola, a Tomaso Messinese, a Giovanni Boccaccio, a Leonardo Beccamuggi, a madonna Laura, et ad altri molti, et eglino a lui. Cose pregiatissime, e rare, et la maggior parte mai più date in luce à propos de laquelle voir Nicola Catelli, " "Li due Petrarchisti”. Un cas d'évolution de la forme dialogue entre le XVI e et le XVII ${ }^{e}$ siècle ", in Les États du dialogue à l'âge de l'humanisme, éd. Emmanuel Buron, Philippe Guérin et Claire Lesage (Rennes-Tours : Presses universitaires de Rennes-Presses universitaires François Rabelais, 2015), 365-374. Consulter également l'édition moderne : Franco, Il Petrarchista... (1979).

16. Luigi Baldacci, Il petrarchismo italiano nel Cinquecento, Nuova edizione accresciuta (Padoue : Liviana Editrice, 1974), 46-48.

17. François Lecercle, "Le Texte comme langue : Cicéronianisme et pétrarquisme ", Littérature 55 (1984) : 45-53.

18. Niccolò Franco, Il Petrarchista... (1539), fol. A iii $\mathrm{r}^{\circ}$ : "Perche saranno molti di quegli, a $i$ quali (per dir cosi) puzzano gli ambragani, che a gran pena c'hauranno accostato il naso, che mi stimeranno incorso nel crime de la lesa maesta del Petrarca. A i quali non intendo rispondere per questa Epistola, per essere l'intento mio presentarui senza contrasti un presentuzzo di carta scritta, al quale, perche non debbia parere cotanto picciolo, farò tosto la giunta d'una Comedia, talche con i ridicoli suggetti uada in parte coprendo 
gaucherie de l'expression de ceux qui vénéraient Pétrarque comme une idole. Lacte de l'édition tournait lui-même en dérision l'acte du courtisan faisant un hommage au seigneur ou à la dame ; Franco le présente ici de manière ironique : cet hommage est non un présent, mais un presentuzzo, un " petit présent » - terme employé en particulier par Castiglione dans son Cortigiano $(1528)^{19}$. Toujours selon l'épître liminaire, Il Petrarchista devait être suivi d'une Comedia : " al quale, perche non debbia parere cotanto picciolo, farò tosto la giunta d'una Comedia... ». Elle ne semble pas avoir été conservée. L’idée lui en venait très probablement de celles de Machiavel ou de l'Arétin ${ }^{20}$.

La Cortigiana de ce dernier dénonçait la manie du pétrarquisme devenue une mode sociale plus qu'un style littéraire ${ }^{21}$. Dès le prologue de la pièce - écrite à Rome en 1525 et éditée avec de nombreuses modifications à Venise en $1534^{22}-$, l'Arétin avait justifié son $\operatorname{propos}^{23}$. La mise en scène qu'il

il riderui de la goffezza del dire. » (« Nombreux seront en effet ceux pour qui, pour le dire ainsi, l'ambre pue, et qui, à peine se seront-ils bouché le nez, m’estimeront tombé dans le crime de lèse-majesté envers Pétrarque. Je n'entends point leur répondre par la présente Épître, car je veux vous présenter sans conflit ce petit présent d'un papier écrit. Afin qu'il ne semble pas trop petit, j'y joindrai bientôt une Comédie conçue de sorte que ses personnages soient si ridicules que votre rire dépasse encore celui suscité par la grossièreté de l'expression »).

19. Reprenant les conseils de l'Ars amandi d'Ovide, il dressait la liste des procédés par lesquels l'amant peut gagner les grâces de l'aimée, parmi lesquels figurait la présentation de petits présents, de lettres, de sonnets : "subito ha apparecchiato un presentuzzo, una lettera, un sonetto, o tal cosa, da darle per parte dello amante ». Vittorio Cian (Baldesar Castiglione, Il Libro del Cortegiano, éd. Vittorio Cian [Florence : Sansoni. 1947], 373) relevait déjà comment Castiglione suivait les conseils d'Ovide, Ars Amandi, lib. II, 261-262 (" Nec dominam jubeo pretioso munere dones : / Parva, sed e parvis callidus apta dato... "), I, 437-438 ("Cera vadum tentet, rasis infusa tabellis : / Cera tuae primum nuntia mentis eat. ») et II, 273-274 ("Quid tibi praecipiam teneros quoque mittere versus? / Hei mihi! non multum carmen honoris habet. / Carmina laudantur; sed munera magna petuntur»).

20. Voir, pour la satire théâtrale du pétrarquiste comme personne, Antonio Stäuble, «Parlar per lettera ». Il pedante nella commedia del Cinquecento e altri saggi sul teatro rinascimentale (Rome: Bulzoni, 1991), 84-115.

21. Piermario Vescovo, « "Col Petrarca in la manica”. Petrarchismo e patologia nella commedia italiana del Cinquecento ", in I territori del Petrarchismo. Frontiere e sconfinamenti, éd. Cristina Montagnani (Rome : Bulzoni, 2005), 171-186.

22. Federico Della Corte. "L'Aretino in tipografia. Preliminari all'edizione della Cortigiana a stampa ", Filologia italiana 2 (2005) : 161-197.

23. Pietro Aretino, Teatro, t. I, Cortigiana (1525 e 1534), éd. Paolo Trovato et Federico Della Corte, intr. Giulio Ferroni (Rome : Salerno, 2010), Prologo, 63 : il s'en prend aux «"sonetti lascivi", "unti", "liquidi 
ébauchait ensuite était celle d'un Parnasse ordonné par Pasquin, qui seul a le pouvoir d'admettre les mots qui sont du règne de la poésie ${ }^{24}$. Plus loin dans la Cortigiana, l'Arétin mettait en scène la conversation entre Maestro Andrea, pédant au latin macaronique, et Messer Maco, prompt à tirer de sa manche un Pétrarque lui servant d'autorité ${ }^{25}$. Après la satire du Pétrarque des courtisans, on avait là celle du Pétrarque des pédants. Au cours des profonds remaniements auxquels il soumit la pièce pour sa réédition en 1534, l'Arétin supprima une partie de ces attaques mais en ajouta d'autres, contre le pétrarquisme caricaturé, non comme manière littéraire mais comme langage mondain ${ }^{26}$.

cristalli", "unquanco", "quinci e quindi" e simili coglionerie, cagion che madonne Muse non si pascono si non d'insalatucce fiorentine " ("sonetti lascivi”, "unti”, "liquidi cristall”, "unquanco", "quinci e quindi” et autres couillonneries de ce genre, qui font que Mesdames les Muses ne s'alimentent que de petites salades de Florence »), mais il justifie aussi l'emploi de mots non florentins, Prologo, 64 : «E se 'l Petrarca non disse thomo, l'ha detto egli ch'è da Bologna et altro omo che 'l Petrarcha per essere eques inorpellato. " («Et si Pétrarque n’a pas dit thomo, le mot a été employé par tel autre [Cinotto], qui vient de Bologne et qui est un homme bien différent de Pétrarque, du fait qu'il est chevalier doré »). Ce qui permet à l'A rétin de conclure un peu plus loin : "Sippa è vocabulo antiquo, deroccare $e$ tartussare modern [i] ; $e$ Cinotto, poeta coronato per man di papa Leon, l'usa : et sta molto bene. Siché, questi comentatori di vocabuli del Petrarca, gli fanno dire cose che non l[e] faria dire al Nocca da Fiorenza viII altri tratti di corda [...]. » («Sippa est un mot ancien, deroccare et tartussare sont des mots modernes, et Cinotto, poète couronné par la main du pape Léon [X], les emploie - et c'est fort bien ainsi. De la sorte, ces commentateurs des vocables de Pétrarque lui font dire des choses que ne ferait pas dire à Nocca de Florence la torture des cordes $[\ldots] »)$.

24. Aretino, Teatro, t. I, Cortigiana (1525 e 1534), 64 : « Non è niuno che sappia meglio di Pasquino quello si può usare o no " ("Personne ne sait mieux que Pasquin ce que l'on peut employer et ce que l'on ne peut pas »).

25. Aretino, Teatro, t. I, Cortigiana (1525 e 1534), acte IV, scène 1, 123 : «Voi mi fate una rima falsa, ché bisognat non è toscano; et ecco qui in la manica el Petrarca che lo conferma» ("Vous me faites une rime éronnée, car bisognat n'est pas toscan, et le Pétrarque que je tiens ici dans la main le confirme »). La version de 1534 maintient dans la même scène une réflexion sur l'emploi d'un mot par Pétrarque, mais en variant ce terme et la langue, Aretino, Teatro, t. I, Cortigiana (1525 e 1534), acte IV, scène 1, 297 : "Messer Maco. Cortigianos no 'l dice il Petrarca. Maestro Andrea. Lo dice in mille luoghi. Messer Maco. È vero, il Petrarca lo dice in quel sonetto È sí debile il filo. » ("MÂ̂Tre Maco. Pétrarque ne dit pas “Cortigianos". Maître André. Il le dit en mille endroits. Maître Maco. C'est vrai, Pétrarque le dit dans le sonnet Ė sí debile il filo»).

26. Aretino, Teatro, t. I, Cortigiana (1525 e 1534), acte I, scène 22, 252 : «MESSER MACo. Come si fa la nimpha? MAESTRo ANDREA. Questo ve lo insegnerà ogni cortigianuzzo furfantino, che sta da un vespro all'altro come un perdono a farsi nettare una cappa et un saio d'accottonato, e consuma l'ore in su gli specchi in farsi i ricci et ungersi la testa antica; e col parlar toscano, e co 'l petrarchino in mano, con un 
C'est cette double veine que Franco suit dans Il Petrarchista et qu'il aurait suivie dans sa Comedia, tournant en dérision non seulement l'adoration vouée à Pétrarque, mais aussi la construction, par Pétrarque, de son propre personnage, destiné à une gloire impérissable, célébrée par de dévots commentateurs - Vellutello ou Gesualdo, en particulier ${ }^{27}$.

Enfin, le choix de Sannio de faire pèlerinage à Vaucluse laisse transparaître une critique de la nouvelle célébrité dont jouissait Avignon depuis que - selon la légende recueillie par Jean de Tournes en 1545 - Maurice Scève y avait retrouvé pour François $\mathrm{I}^{\mathrm{er}}$ le tombeau de Laure. L'événement avait donné lieu à un véritable pèlerinage littéraire, dont le premier à donner l'exemple avait été François I ${ }^{\text {er }}$ lui-même. Dans le tombeau de Laure aurait aussi été retrouvé un sonnet inédit consacré à Laure, qui devint l'objet de nombreux commentaires ${ }^{28}$.

C'est pourquoi Franco, dans le récit du voyage en Avignon, parodia l'attitude philologique de ses contemporains et offrit à ses lecteurs des pastiches de lettres de Pétrarque et de Boccace, dans lesquelles il révélait des aspects méconnus de l'amant de Laure. La parodie du fétichisme antiquaire le mena jusqu'à faire découvrir à Sannio, l'alter ego de Niccolò Franco, outre l'« effigie » de Laure - son portrait peint par Simone Martini, que Pétrarque évoquait dans les sonnets Rvf 77 et 78 -, des " choses de plus haute importance » (« cose di maggior importanza »), dans une liste burlesque couronnée par un curedents et de nombreux fragments d'un pot de chambre (" molti fragmenti di un orinale $")^{29}$. La parodie ne porte pas seulement sur le caractère dégradant de l'objet qui remplace les objets communément associés au culte de la bien-aimée,

\footnotetext{
"sí, a fe", con un "giuro a Dio", e con un "bascio la mano" gli pare essere il Totum Continens. " (" MAître Maco. Comment fait-on le damoiseau ? MaÎTre André. «Vous l'apprendrez du premier faquin de courtisan qui, entre vêpres et vêpres, est à nettoyer comme une patène sa cape et son pourpoint de drap frisé, et passe des heures devant les miroirs à boucler ses cheveux et à oindre sa vieille tête ; et qui, avec son parler toscan et ses citations du "Petit Pétrarque", avec son "sì, a fe", avec son "giuro a Dio" et avec son "bascio la mano", se croit être le Totum continens").

27. Voir Roberto L. Bruni, « Parodia e plagio nel Petrarchista di Nicolò Franco ».

28. Voir Verdun-Louis Saulnier, "Maurice Scève et l'épitaphe de Laure », Revue de littérature comparée 24 (1950) : 65-78, Enzo Giudici, "Bilancio di una annosa questione : Maurice Scève e la "scoperta" della "tomba di Laura". » Quaderni di filologia e lingue romanze 2 (1980) : 3-70, et Olivier Millet, « Le Tombeau de la morte et la voix du poète : La mémoire de Pétrarque en France autour de 1533 », in Regards sur le passé dans l'Europe des XVI et XVII siècles, éd. Francine Wild (Berne : Peter Lang, 1997), 183-195.
}

29. Niccolò Franco, Il Petrarchista... (1539), fol. $18 \mathrm{v}^{\circ}$. 
elle se laisse aussi déceler dans l'emploi du mot «fragmenti », essentiel dans la poésie de Pétrarque depuis le titre même du Canzoniere : Rerum vulgarium fragmenta. Plus loin - nouvelle parodie de la philologie pétrarquiste que Bembo avait encouragée par l'examen des manuscrits du poète - , on découvre une série de versions originales de poèmes de Pétrarque. Initialement, il n'avait pas écrit Benedetto sia'l giorno, e'l mese, e l'anno (Rvf 61), mais Maladetto sia il giorno, e'l mese, e l'anno... ; non pas Io ho pregato amore, e nel riprego... (Rvf 240), mais Io n'ho 'ncacato amore, e gliene incaco ; non pas Passato e'l tempo homai lasso, che tanto... (Rvf 313), mais Passato e'l tempo ; che Berta filava; non pas Per fare una leggiadra sua vendetta (Rvf2), mais Per farmi amor nel buco una borsetta; non pas, enfin, Di pensier en piensier, di monte in monte (Rvf 129), mais Di bordell' in bordel, di chiasso in chiasso / Mi guida amor ${ }^{30}$. Ce ne sont là que quelques-unes des trouvailles burlesques de Franco, qui s'adonne à la parodie du pétrarquisme, comme avant lui Berni, Molza et l’Arétin.

\section{Le canon poétique méridional de Franco}

Si cette inspiration des modèles burlesques a été relevée, il convient de rechercher, par-delà l'évident plaisir qu'il prenait à rire de la mode du pétrarquisme sous des airs d'Érasme italien, les motivations de Franco. Il connut longtemps la réputation d'un Zoïle, d'un critique médisant emporté par sa fureur ${ }^{31}$. Il a été montré combien Franco exerça sa verve satirique dès ses années dans la capitale parthénopéenne, s'en prenant en particulier à Gianio Anisio ou à Girolamo Borgia $^{32}$. Dans cette ligne, il convient de considérer que, si Franco s'en prend à certains auteurs méridionaux, c'est qu'il vient de leurs cercles, où il a fait ses premières armes; ce qui explique aussi sa distance à l'égard du modèle pétrarquiste de Bembo et de ses imitateurs.

30. Niccolò Franco, Il Petrarchista... (1539), fol. 22r ${ }^{\circ}-23 \mathrm{r}^{\circ}$.

31. Franco était ainsi présenté dans la Piazza universale de Tommaso Garzoni comme le plus médisant des critiques - le médisant par excellence, plus encore que l'Arétin. Cette réputation se diffusa dans toute l'Europe ; voir Roland Béhar, "Homeromastix, Vergiliomastix... ¿Gongoramastix? », e-Spania 18 (2014) : https://e-spania.revues.org/23769 et Marco Faini, " “E poi in Roma ognuno è Aretino”... ».

32. Jesús Ponce Cárdenas, «Ma io son pure napolitano: Nicolò Franco e i circoli meridionali (15311543) ", in Rinascimento meridionale. Napoli e il viceré Pedro de Toledo (1532-1553), éd. Encarnación Sánchez García (Naples : Tullio Pironti, 2016), 203-234. 
La bibliothèque poétique idéale qu'il décrivit dans le huitième de ses Dialoghi piacevoli démontre que son canon était encore tout napolitain ${ }^{33}$. Le catalogue des poètes à imiter, qui commençait avec Sannazar et culminait dans l'évocation d'une triade d'amis - Epicuro, Rota et Tansillo - , n'était aucunement conforme à l'orthodoxie bembienne. Le seul des auteurs qu'il célèbre qui soit décédé à cette date est Sannazar, mort en 1530, ce qui rend sa mention d'autant plus significative. Bembo, en revanche, est absent. Une autre épître, adressée à sa lampe, donne lieu à une longue réponse de celle-ci (Risposta della lucerna) : elle y évoque une suite de poètes où Bembo apparaît sous les traits du législateur, et la liste culmine à nouveau dans la triade des amis de Franco : Epicuro, Rota et Tansillo ${ }^{34}$. Dans une autre épître encore, Franco s'en prend avec la plus grande virulence à Girolamo Borgia, un " pédant " napolitain se réclamant des mêmes cercles pontaniens. Il retourne contre lui tous les lieux communs de la critique humaniste des grammairiens, formulés en

33. Niccolò Franco, Dialoghi piacevoli (Venise : Antonio Gardane, 1542), fol. $\mathrm{CXr}^{\circ}$ : «E se pur voi tenere [des livres de poésie], fa che sieno le cose come sarebbe a dire d'un Sannazaro, d'un Molza, d'un Alamanni, d'un Varchi, d'un Bevezzano, d'un Epicuro, d'un Rota, e d'un Tansillo, perché questi sono, e saranno i veri poeti, et il loro si può dir poetare e non trafuggare. " ("Et si tu veux avoir des livres de poésie, fais en sorte qu' ils soient pleins de vers que l'on dirait d'un Sannazar, d'un Molza, d'un Alamanni, d'un Varchi, d'un Bevezzano, d'un Epicuro, d'un Rota et d'un Tansillo, car ce sont là, et ce seront toujours, les poètes véritables, et leurs vers peuvent être dits de poésie, et non de larcin. »). On peut également consulter l'édition des Dialoghi piacevoli, éd. Franco Pignati (Manziana : Vecchiarelli, 2003); voir Emilio Speciale, « Nicolò Franco, il libraio e la letteratura », Schifanoia 10 (1990) : 175-187.

34. Niccolò Franco, Le Pistole Vulgari di M. Nicolo Franco (In Vinetia nele Stampe d'Antonio Gardane, 1539), fol. LXXXVIv : " Veggo nel sommo loro il Bembo, il quale, come ottimo, e massimo Duce di tutti gli altri, si sta dando ordini, e leggi con lo scettro de la scienza, minacciando prigion d'infamia, e morte di nome a chi non osserva i giusti decreti de la sua penna. Gli veggo apresso i due Girolamo, l'un Quirino, e l'altro Molino. Veggoci i due Bernardi, l'un Navaiero, e l'altro Cappello. Questi non mai satii di star con riverenza inanzi la Maesta del venerando Vecchio, tutta via attentamente esseguiscono cio che egli ordina di sua bocca. Veggo anche nel medemo collegio, il Molza, il Fortunio, lo Spirone, il Bevazzano, il Gratia, il Tasso, l'Alemanno, il Varchi. Veggoci il mio Epicuro, il Rota, il Tansillo." ("Je vois à leur tête Bembo, qui, comme meilleur et premier chef de tous les autres, répartit les ordres et les lois avec le sceptre de la science, menaçant de la prison d'infamie et de la mort du nom ceux qui n'observent point les justes décrets de sa plume. Près de lui, je vois les deux Girolamo, Quirino l'un, Molino l'autre. Je vois les deux Bernardo, Navagero l'un, Cappello l'autre. Ceux-ci ne sont jamais assez contents d'adorer avec révérence la Majesté du vénérable Vieux, et ils exécutent avec diligence ce qu'il ordonne de sa bouche. Dans ce même collège, j'aperçois aussi Molza, Fortunio, Sperone, Bevezzano, Gratia, Tasso, Alamanni, Varchi. Et j'y vois encore mon cher Epicuro, Rota et Tansillo»). 
particulier par Pontano dans le Charon $^{35}$. Le Parnasse poétique s'organise dans cette épître de manière concentrique, autour de Pontano, suivi de Sannazar, de Carbone, de Summonte et de Gravina, "fiori degli intelletti ». Si Borgia veut guérir de sa folie, il lui faut laisser les mauvais grammairiens - Scoppa, au premier chef - et retrouver la bonne inspiration, qu'il trouvera auprès de Marcantonio Epicuro, de Rota, de « son » Tansillo ( [i]l mio Tansillo ») et de Philocalo. Là encore, les bons auteurs étaient ceux de l'académie pontanienne et de leurs héritiers vernaculaires. Cette vision de la poésie de son temps, qui sous-tend l'ensemble de ses jugements sur ses contemporains, Franco la doit à sa formation napolitaine ${ }^{36}$. Or, la critique de Bembo avait elle-même été

35. Niccolò Franco, Le Pistole Vulgari... (1539), fol. XCXVIr ${ }^{\circ}-\mathrm{v}^{\circ}$ : « Gli huomini non ponno essere tutti pari, ne tutti i pedanti tornar Pontani. Non è impresa d'ogni braghiere farsi il Sannazaro; et non è somma d'ogni bestia diventare il Carbone, o il Summontio, o il Gravina, fiori degli intelletti. Non è la meglior cosa per voi che fare versacci ad otto et a nove piedi come le piattole. Il fatto vostro è accomodarvi a la libera. Non sta bene al vecchio andare stringato, ch'una stringa che gli tenga il braghetto gli basta assai. Elegantie a chie ne vole. Colori, argutie a chi è giovane e puote, e non a te, vecchio, che non hai forza ne la balestra. Sì che Borgio, fa a senno mio che senza uscir altrimenti di Napoli, t'insegnarò un secreto, che beverai del Cavallino cosi asino come sei. Non accade gran manefattura a quel che voglio io, non haverai a fare altro se non lasciare la prosuntione di banda, reintegrarti ne l'amicizia degli huomini degni: adorare principalmente il dotto Epicuro, farti schiavo del nobil Rotilio e del mio Tansillo, forbire il culo al Philocalo, poiché non sei buono ch'egli lo netti a te; lasciar le sette che vai facendo con le carogne degli Scoppi, dei Basilii, dei Cesarii e di simili gentuzze. Accostati con quegli s'hai voglia di spogliarti del bue, che t'ammanta. » ("Les hommes ne peuvent pas être tous égaux, et tous les pédants ne peuvent devenir des Pontano. Il n'est en effet pas à la portée de tout brayer de faire comme Sannazar, et toute bête de somme ne devient pas Carbone, Summonte ou Gravina, fleurs des entendements. Vous ne savez faire autre chose que de méchants vers de huit ou neuf pieds, comme les morpions. Vous avez pour coutume de vous arranger à votre guise. Il ne sied pas au vieil homme d'être enserré, car une ficelle qui lui tient la ceinture est déjà bien suffisante. Les élégances, pour qui les veut. Les couleurs, les pointes, pour qui est jeune et peut, et non pour toi, vieillard, qui n'as ni force, ni arbalète. Ainsi, Borgia, fais en sorte de suivre mon conseil. Sans sortir autrement de Naples, je vais t'enseigner un secret, car tu boirais de la jusquiame, âne que tu es. Point besoin d'un grand artifice, pour ce que je conseille : il te suffira de laisser de côté la présomption et d'entrer à nouveau dans l'amitié d'hommes dignes, d'adorer avant tout le docte Epicuro, de devenir l'esclave du noble Rota et de mon cher Tansillo, de torcher le cul à Philocalo, car tu ne vaux pas qu'il te le nettoie à toi, et enfin d'abandonner les sectes que tu formes avec ces charognes de Scoppa, de Basilio, de Cesare et d'autres gens peu recommandables. Rapproche-toi de ceux-là si tu désires te défaire du veau qui te recouvre. " «Beverai del Cavallino » est un probable jeu de mot sur la jusquiame, "dente di cavallino", et la source des Muses, Hippocrène, source « chevaline » créée par Pégase).

36. Amedeo Quondam, dans un riche essai sur la portée d'Il Petrarchista, voit dans ce canon le pari d'un Franco désireux de promouvoir sur la place de Venise ses amis napolitains, dont on peut 
formulée à Naples, au fil des réflexions sur la questione della lingua autant que dans la pratique poétique, en particulier dans l'œuvre de Tansillo.

\section{La réception de Bembo à Naples}

Dès leur publication, les Prose della volgar lingua (1525) de Pietro Bembo avaient été accueillies avec une relative froideur dans les cercles napolitains, qui mettraient plusieurs décennies avant de céder au succès du pétrarquisme. Alors qu'il appréciait Bembo, Sannazar confiait à Paul Jove qu'il eût préféré qu'il ne publiât pas les Asolains, et que sa Grammaire - les Prose - lui semblait scrupuleuse, âpre et affectée, dénuée de la délicatesse dont faisaient preuve ses sonnets, ses stances et ses vers latins ${ }^{37}$. Jove fit sien cet avis dès $1527:$ au Sannazar poète du De Partu Virginis, des Eclogee piscatorice et du lusus latine et vernacule -, il opposa un Bembo " Novus Aristarcus ", "Grammaticæ conditor ", "Alter Priscianus " de la langue vulgaire ${ }^{38}$. Naples fut longue à accepter le pétrarquisme tel que le XVI ${ }^{\mathrm{e}}$ siècle, à la suite de Bembo, l'adopta et le pratiqua. Dans les années 1520 et 1530, nombreuses furent les critiques de ce langage nouveau que le Vénitien imposait aux cours, cependant que d'autres Vénitiens, au premier chef le divin Arétin, le prenaient à partie.

déduire « l'engagement de la part de Franco pour devenir le promoteur, auprès des éditeurs et des lettrés vénitiens, des écrivains napolitains, mais aussi, du fait de l'emphase et de la précocité de la reconnaissance des trois, la fonction instrumentale que Franco leur attribue dans l'économie polémique de son argumentation satirique et parodique, afin de produire un effet de contraste tant par rapport aux Florentins de son temps qu'il évoque dans les dernières lignes de sa lettre à Pétrarque que par rapport à la domination alors exercée par les cicéroniens (vénétiens et bembiens) larrons de petits mots pétrarquesques. » (Amedeo Quondam, « Petrarchisti e gentiluomini. 2. Ladri di parolette: per non essere mai più Tebaldei ", in Petrarca. Canoni, esemplarità, éd. Valeria Finucci [Rome : Bulzoni, 2006], 21-71, ici 39).

37. Voir Paul Jove, dans une lettre à Scannapeco (1535), Paulo Giovio, Opera, t. 1 : Lettere, éd. Giuseppe Guido Ferreo (Rome : Istituto Poligrafico dello Stato - Libreria dello Stato, 1956), 177 : «Non mi disse egli [i.e. Sannazar] del suo tanto amato Bembo ch'el vorrebbe che non avesse dato fuora gli Asolani? E che la Grammatica gli pareva scrupulosa, aspra e affettata e non simile alla delicatura dell'ingegno del Bembo, tanto divino ne' sonetti e stanze, e ne' versi latini tanto adorato e candido? " ("Sannazar ne m'a-t-il pas dit qu'il aurait préféré que son très cher Bembo ne publiât pas les Asolains ? Et que la Grammatica lui semblait pleine de scrupules, âpre et affectée, très peu ressemblante à la délicatesse d'esprit de Bembo, si divin dans ses sonnets et dans ses stances, si admiré et pur dans ses vers latins? »).

38. Paul Jove, Dialogus de viris et foeminis aetate nostra florentibus (1527), cité d'après Carlo Vecce, «Paolo Giovio e Vittoria Colonna », Periodico della Società Storica Comense 54 (1990) : 67-93, ici 76. 
Il faut en réalité attendre la fin de la décennie 1530 pour voir s’imposer le modèle de Bembo. L’opuscule inédit Intorno alla volgar lingua, composé par Luca Peto vers 1538, reposait entièrement sur la doctrine des Prose ${ }^{39}$. Et ce sont surtout les Regole osservanze, et avvertenze sopra lo scrivere correttamente la lingua volgare Toscana in prosa et in versi du fuoruscito florentin Paolo del Rosso, publiées à Naples par Matteo Cancer en 1545, qui y affirmèrent le rôle central de Bembo dans la définition de l'art d'écrire en langue toscane ${ }^{40}$. Ce mouvement se prolonge par la publication des Prose di Monsignor Bembo ridotte a metodo (1569) de Marco Antonio Flaminio (mort en 1550). L'opuscule réduisait à un vaste vocabulaire la doctrine bembienne des Prose. Rédigées sans doute encore avant l'arrivée de Flaminio à Naples en 1538, ces Prose « ridotte a metodo " y avaient contribué à diffuser les normes lexicales et grammaticales de Bembo ${ }^{41}$.

Face à cette ligne de l'« orthodoxie » destinée à s'imposer progressivement, une autre s'attacha à défendre, contre la suprématie de Pétrarque, la légitimité de l'imitation d'autres modèles, tels Dante, Sannazar, l'Arioste ou, même, Machiavel et l'Arétin. Après la mort de Sannazar, en l'absence d'une autorité littéraire supérieure, Naples demeura un temps méfiante. Des travaux plus récents ont mis en lumière le mouvement de résistance à Bembo, dû autant à l'attachement à des autorités littéraires plus anciennes - ainsi, pour Fuscano, celle d'un Cristoforo Landino, commentateur de Dante ${ }^{42}$ - qu'à l'admiration pour un Sannazar disparu en 1530. Cette tendance, plus circonstanciée, s'accordait mieux avec les conceptions des poètes napolitains d'alors : Luigi

39. Voir Pasquale Sabbatino, «L'inedita grammatica di Luca Peto », in Il modello bembiano a Napoli nel Cinquecento (Naples : Ferraro, 1986), 83-101.

40. Voir Pasquale Sabbatino, « Le "regole" del fiorentino Paolo del Rosso nella "nobilissima cittade" di Napoli ", in L'idioma volgare. Il dibattito sulla lingua letteraria nel Rinascimento (Rome : Bulzoni, 1995), 131-147 et « "Per ragione di grammatica”. Le Prose del Bembo, Il Polito del Tolomei e le Regole di Paolo del Rosso », in L'idioma volgare. Il dibattito sulla lingua letteraria..., 149-238.

41. Voir Pasquale Sabbatino, «L'inedita grammatica di Luca Peto ».

42. Giovanni Berardino Fuscano, Della oratoria e poetica facoltà, publié dans ses Stanze sovra la bellezza di Napoli (Rome : Blado d’Asola, 1531), éd. Cristiana Anna Adesso (Naples : Edizioni scientifiche italiane, 2006). 
"Il ridervi de la goffezza del dire" : Niccolò Franco et la satire napolitaine du pétrarquisme 201

Tansillo, en particulier, mais aussi Bernardo Tasso, qui loua Antonio Brocardo pour sa progressive distanciation du modèle poétique bembien ${ }^{43}$.

Par ailleurs, les théoriciens du vers et de la langue, tels Benedetto Di Falco ou Fabrizio Luna, auteurs du Rimario (1535) et du Vocabulario (1536), et même certains commentateurs de Pétrarque, tel Sylvano de Venaphro, auteur en 1533 d'Il Petrarcha, manifestaient leurs réticences à adopter les normes et les règles énoncées dans les Prose.

Les opinions de ces derniers ont été mises en lumière par Carlo Dionisotti, mais le jugement qu'il porta sur leurs textes dès qu'il les eut donnés à connaître greva leur réception. On se souvient souvent des mots par lesquels Sylvano de Venaphro ouvrait son Petrarca, où il demandait à Bembo de pardonner ses manquements aux règles qu'il avait définies dans les Prose ${ }^{44}$. Lattitude du commentateur pouvait - comme le fit Dionisotti ${ }^{45}$ - être mise au compte

43. Voir Erika Milburn, Luigi Tansillo and Lyric Poetry in Sixteenth-century Naples (Leeds : Maney Publishing for the Modern Humanities Research Association, 2003).

44. Sylvano de Venaphro. Il Petrarca con commento di Sylvano da Venafro (Naples : Ant. Jovino et Matthio Canzer, 1533), fol. $1 \mathrm{r}^{\circ}$ : «Prego solamente il mio Bembo, non li dispiaccia di perdonarmi se non ho osservato tutto quel ch'egli scrive della volgar lingua al suo Medici, ch'io ci ho faticato assai, ma non si può, da un nato nelle selve e nutrito, senza gran tempo diventar toscano. Né gli orecchi m'han voluto assentire ch'io dica chenti, altresì, guari, sapiendo, guatare, conchiudere, testé, perché, oggimai, con alcune altre voci che'l mio odito rifuge. In questo so che si potrà tener ben servito da me, che, qualor non mi è uscito di mente, ho detto agevole a suo modo e non facile, malagevole e non difficile, lui $e$ lei negli obliqui e loro. $\dot{E}$ ben vero ch'io mi sono alle volte scordato e ho detto lui e lei nel primo caso, col verbo sustantivo. Facciami intender che pena ne va, ch'io ci la inviarò fin a Venezia. Ma io so ch'egli è molto cortese e, secondo intendo, di tai proventi ne fa poco caso e ne suol donar la maggior parte, il che fa da suo pare, perché oltre di esser il più famoso e scienziato nelle discipline di liberali, dicono esser ricco e di molta nobiltà. " (" Je prie seulement mon cher Bembo de bien vouloir me pardonner si je n'ai pas observé tout ce qu'il écrit à son cher Médicis à propos de la langue "vulgaire" ; je m’y suis assez efforcé, mais il est impossible en peu de temps de devenir toscan à quelqu'un de né et de nourri dans les bois. Mes oreilles ne m’ont pas permis de dire chenti, altresì, guari, sapiendo, guatare, conchiudere, testé, perché, oggimai, ainsi que quelques autres mots que mon ouïe fuit. En cela, je sais qu'il pourra se tenir pour bien servi de ma part, moi qui tant que je ne l'oubliais pas, ai dit agevole à sa manière, et non facile, malagevole, et non difficile, lui et lei dans les cas obliques, et loro. Certes, j'ai parfois oublié et ai dit lui et lei dans le premier cas, avec le verbe substantif. Qu'il me fasse savoir quelle peine j'encours, et je la lui envoie jusqu'à Venise. Mais je sais qu'il est très courtois et, selon ce que je pense, de revenus de ce genre il ne fait guère de cas : il a pour coutume, de son propre chef, d'en donner la plus grande part, car il n'est pas seulement le plus renommé et le plus docte dans les disciplines libérales, il est aussi, dit-on, riche et de grande noblesse »).

45. Carlo Dionisotti, «Appunti sulle Rime del Sannazaro », Giornale storico della letteratura italiana 140 (1963) : 161-211 (rééd. in Scritti di storia della letteratura italiana, éd. Tania Basile, Vincenzo Fera et 
de son provincialisme, de sa rusticité, qui n'étaient en effet pas seulement rhétorique. Mais pourrait-on en dire autant de Fabrizio Luna, l'auteur du Vocabulario (1536), ou de Benedetto Di Falco, dont le Rimario (1535) constitua un moment majeur dans le développement de la lexicographie italienne au $\mathrm{XVI}^{\mathrm{e}}$ siècle et inspirerait à Ruscelli son rimario, Del modo di comporre in versi nella lingua italiana (1558) ?

On esquissera, dans ce qui suit, la vision que Luna et Di Falco avaient de Bembo et du pétrarquisme, afin de montrer comment elle préparait, sans en développer toutes les possibilités, la critique du Petrarchista de Franco ${ }^{46}$. Sans affirmer que Franco leur devait ses idées, sa comparaison avec ses prédécesseurs montrera le type de satire littéraire qu'il connut à Naples, dont il allait s'inspirer pour mieux s'en distancier et l'adapter aux formes littéraires plus proprement vénitiennes.

\section{Fabrizio Luna et l'ouverture du canon poétique}

Fabrizio Luna est l'exact contemporain de Di Falco et de Franco ${ }^{47}$. Il publie en 1536 son Vocabulario di cinquemila vocabuli toschi non men oscuri che utili e necessarii del Furioso, Boccaccio, Petrarca e Dante novamente dichiarati e raccolti [...] per alfabeta ad utilità di chi legge, scrive e favella, à Naples, chez Sultzbach. Disciple de Pietro Summonte, ami de Dragonetto Bonifacio, membre du cercle autour de Vittoria Colonna, il compose une série de poèmes néo-latins, le Sylvarum, elegiarum et epigrammatum libellus (1534), aujourd'hui

Susanna Villari [Rome : Edizioni di Storia e Letteratura, 2009], II. 1-37), ici, 203 : « Ils portaient dans leurs œuvres, sans s'en rendre compte et sans pudeur non plus, la simplicité d'une éducation dialectale et barbare que l'on aurait eu du mal à trouver ailleurs, aux mêmes dates ».

46. Ces deux esquisses reprennent le fil d’un travail commencé dans Roland Béhar, «La questione della lingua dans les cercles académiques napolitains du XVI e siècle ", in Villes à la croisée des langues (XVI ${ }^{e}$ XVII siècles) : Anvers, Hambourg, Milan, Naples et Palerme, coord. Roland Béhar, Mercedes Blanco et Jochen Hafner, Genève : Librairie Droz, sous presse.

47. Milena Montanile, "Il Vocabulario di Fabricio Luna ", in La parole e la norma: studi su lessico e grammatica a Napoli tra Quattro e Cinquecento (Naples : Edizioni Scientifiche Italiane, 1996), 47-74 et Tobia R. Toscano, "Due "allievi" di Vittoria Colonna : Luigi Tansillo e Alfonso d'Avalos ", in Letterati corti accademie. La letteratura a Napoli nella prima metà del Cinquecento (Naples : Loffredo Editore, 2000), 105-108. 
perdu, que Niccolò Franco justement attaqua ${ }^{48}$. Luna lui-même fut d'un esprit acerbe, prompt à la critique - ainsi lorsque, dans son Vocabulario, il reprocha à Gesualdo ses emprunts à Fausto da Longiano ${ }^{49}$.

Le canon des poètes dont il s'inspire est ample et varié, même si l'essentiel des termes du Vocabulario est prélevé chez Dante, Pétrarque, Boccace, l'Arioste, l'Arétin, Sannazar et Bembo. Il n'exclut pas d'autres noms et il reprend même à deux de ses auteurs, Castiglione (Courtisan, I, v) et l'Arétin (Il Marescalco, V, III, 5), des listes des bons auteurs à suivre, dont le dernier est précisément Di Falco, revendiqué comme Napolitain ${ }^{50}$.

De manière plus générale, on peut dire qu'à l'exception de l'Arioste et de l'Arétin, il va puiser ses sources chez des poètes " méridionaux ", de Sannazar à Tansillo. Ses goûts sont pour l'essentiel ceux de Franco lorsque celui-ci quitte Naples pour Venise, la même année 1536, et lui-même rêve d'effectuer un périple de la Péninsule, qu'il décrit dans l'adresse finale à son livre, annonçant qu'il voyagera jusqu'à la Sérénissime, où il trouvera « il dotto Bembo, il sagace Divino et il degno Ludovico Dolcio, chiari lumi dela nostra volgar lingua $»^{51}-$ alors que le Bembo poète est complètement absent du Vocabulario, de même que celui des Asolani ${ }^{52}$.

On a pu relever dans le livre quelques traits de critique anticléricale, qui manifestent aussi la connaissance d'auteurs germaniques (Eobanus Hessus et Ulrich von Hutten), ce qui répondrait à l'érasmisme d'un Franco qui maîtrisait les écrits du maitre de Rotterdam avant même d'arriver à Venise.

Mais c'est surtout l'intérêt pour l'Arioste et l'Arétin qui retient l'attention, outre celui pour Sannazar. L'Arioste est grandement admiré dans la Naples des années $1530^{53}$. Luna voit en lui l'auteur de l'Énéide furieuse, faisant ainsi de lui un nouveau Virgile, et on a récemment retrouvé des compositions néolatines

48. Voir Antonio Altamura, "Fabrizio Luna e due invettive inedite di Niccolò Franco », Samnium 23 (1950) : 100-105 et Jesús Ponce Cárdenas, « Ma io son pure napolitano ».

49. Voir Gino Belloni, Laura tra Petrarca e Bembo.

50. Voir Pasquale Sabbatino, « La Grammatica della letteratura volgare a Napoli nel Cinquecento », in L'idioma volgare. Il dibattito sulla lingua letteraria..., 13-74, ici 59-60.

51. Tobia R. Toscano, « Due “allievi” di Vittoria Colonna...», 119.

52. Voir Erika Milburn, «La biblioteca di Fabrizio Luna : nell'officina di un lessicografo del Cinquecento », Letteratura italiana antica 8 (2007) : 425-458.

53. Tobia R. Toscano, «Due “allievi” di Vittoria Colonna... ». 
napolitaines inédites célébrant le poète de Ferrare au moment de sa mort ${ }^{54}$. C'est que l'Arioste offrait aussi, pour qui s'intéressait à la richesse de l'expression poétique, l'une des œuvres les plus originales et novatrices par son classicisme même, issu d'une variation subtile sur le langage pétrarquiste. Face à un code bembien refermé sur lui-même, la poésie de l'Arioste représentait l'ouverture à la variété ${ }^{55}$. L'un des rares moments où Bembo apparaît, c'est lorsque Luna reprend un passage des Prose della volgar lingua (II, XVIII) pour préférer comme modèle l'Arioste à Dante ${ }^{56}$.

Outre l'Arioste, l'Arétin trouvait une place particulière dans le Vocabulario de Luna : avant tout pour la Marfisa, son poème épique, pour ses œuvres pieuses et, en même temps, pour ses Ragionamenti et ses pièces de théâtre publiées entre 1533 et 1535 : Il Marescalco, auquel Luna reprend en partie son catalogue des auteurs, et La Cortigiana, dont on a vu le rôle dans la stylisation du personnage du pétrarquiste avant même sa création officielle en 1539, dans le Petrarchista de Niccolò Franco.

Par ces choix, Luna rejoint fortement ceux de Di Falco, qui publia son Rimario l'année antérieure et que Franco avait sans doute bien mieux connu encore que Luna, au point, peut-être, de voir en Luna un rival alors que Di Falco pouvait déjà apparaître en maître.

54. Roland Béhar, « Garcilaso de la Vega et la hiérarchie des genres poétiques. Remarques sur l'Églogue II et l'imitation de l'Arioste ", in La Renaissance des genres, Pratiques et théories entre Italie et Espagne $\left(X V^{e}-X V I I^{e}\right.$ siècles), éd. Paloma Bravo et Giuseppe Sangirardi (Dijon : Éditions Universitaires de Dijon, 2012), 111-122.

55. Selon Stefano Jossa, La Fantasia e la memoria. Intertestualità ariostesche (Naples : Liguori, 1996), 76-77, pour l'Arioste, « Pétrarque garantit la modernisation linguistique des modèles classiques, cependant que les modèles classiques permettent d'éviter que la littérature — et la langue poétique — ne se réduisent au seul Pétrarque, à un jeu de répétition et de reproduction d'un même modèle, d'un même mécanisme. C'est là, en définitive, la grande distance entre l'Arioste et Bembo : face à la norme des Prose della volgare lingua, qui définit un code fermé, exclusif et immuable, caractérisé par le primat de la section lyrique sur tout autre type de langage, la langue du Furioso apparaît fondée sur un équilibre entre Pétrarque et les autres modèles, en particulier les classiques latins, qui sont les seuls qui soient en mesure d'empêcher la maniera tout en évitant la transgression ». Cf. en outre Marco Praloran, « Petrarca in Ariosto : il principium constructionis ", in Lingue del racconto : studi su Boiardo e Ariosto (Rome : Bulzoni, 2009), 175-198.

56. Erika Milburn, « La biblioteca di Fabrizio Luna ... », 442. 


\section{Le Rimario de Benedetto Di Falco, le pétrarquisme et le Testament du porc}

Le Rimario (1535) ${ }^{57}$ de Benedetto Di Falco prend modèle sur dix « autori della lingua volgare »: Pétrarque, Dante, Boccace, Arioste, Pulci, Sannazar, Bembo, Machiavel, Landino et Castiglione. Ce faisant, il se libère de l'emprise du modèle unique $^{58}$. Vers la fin du Rimario, on trouve quelques considérations sur l'état de la langue italienne, que Di Falco souhaiterait " commune ». Ces considérations se prolongent ensuite en une défense contre le reproche qui lui a été adressé de ne pas s'en tenir à la norme de Pétrarque et s'achèvent par un éloge d'un poète ami, également membre des cercles de l'Académie pontanienne, Bernardino Martirano ${ }^{59}$.

Sa défense est amenée avec art. Reprenant celle de saint Jérôme dans son introduction à son commentaire à Isaïe, Di Falco se présente en gentilhomme courtois que la médisance des pédants vient troubler dans sa sage et studieuse retraite. Il est insupportable, alors qu'on écrit tranquillement, en quête de la science des étoiles, de devoir répondre aux objections des éclopés (sciancati) plutôt qu'à celles des hommes de science (scientiati). C'est, écrit Di Falco, comme l'âme qui, au lieu de s'élever vers sa condition angélique, se souvient des turpitudes humaines, tel le prêtre qui, lorsqu'il revêt les vêtements du sacerdoce, affronte tous les vices. Cependant, il peut aussi advenir que l'homme pris dans la mêlée de la guerre découvre en lui-même sa noblesse naturelle, sa cortesia et sa creanza. Les lettres tempèrent les armes, mais les armes mettent

57. L’ouvrage est dédié à un ensemble d'esprits raffinés dans les cercles napolitains desquels on discutait de poésie : Fabrizio Gesualdo, son frère Camillo, archevêque de Conza, Antonio Doria, neveu d'Andrea Doria, Raimondo Orsini, comte de Piacentro - à l'épouse duquel Di Falco dédia peu après son Trattato di amore (Naples : Sultzbach, 1538), Scipione Capece, principal héritier de l'Académie pontanienne après la mort de Sannazar, et enfin Antonio da Gagliano.

58. En 1539, Di Falco consacre même un bref ouvrage à la Dichiaratione de' molti luoghi dubbiosi d'Ariosto, e d'alquanti del Pet[rarca]. Escusation fatta in favor di Dante per Benedetto di Falco Napolitano al Virtuoso Giovane Fabrizio da Gagliano Fiorentino (s.l., s.d.).

59. Il fait notamment l'éloge du poème aujourd'hui perdu de celui-ci sur les amours d'Hysmine et Hysminias. Le Rimario de Di Falco entretient des liens forts avec l'œuvre de B. Martirano et, plus généralement, la poésie néolatine napolitaine ; voir Tobia R. Toscano, « Bernardino Martirano tra pratica del volgare e tradizione pontaniana », in Letterati Corti Accademie. La letteratura a Napoli nella prima metà del Cinquecento (Naples : Loffredo, 2004), 265-298 et Roland Béhar, " La questione della lingua dans les cercles académiques... ». 
la doctrine des lettres à l'épreuve. Médire n'est pas un acte chevaleresque (" maledire che non è atto cavalleresco ») et un sage ne peut s'en prendre à un autre sage, comme Di Falco l'a écrit à propos de Dante et de sa descente aux Enfers. Sur ces considérations, Di Falco en vient à la cause de ses propos. D’infâmes pédants, des huominucci, lui ont reproché sans vergogne d'avoir cité dans son Rimario trop d'autorités littéraires à imiter. Ces pédants se discréditent par leurs manières, courtisant les mères de leurs élèves par leurs protestations empruntées d'amants éplorés ("fingendo esser fedeli amanti, sonettando dela fronte dela luna, de capei d'oro, de denti eburnei, l'avorio, le perle, madonna, segnora, che siete bianca come un cigno, \& altre parole simili $\left.{ }^{60}\right)$. Par leur fétide cuistrerie ("con lor pedentesca fetidita»), ces pédants prétendent s'élever à la hauteur des divins esprits des doctes. De même, un Poète des plus rares et des plus excellents est galvaudé sur les lèvres de certains ociosi, qui se font appeler - on ne saurait dire à quel titre - cavalieri. Imbus d'eux-mêmes, ils jouent les jaloux auprès de leurs amantes au moyen d'amorose paroluzze que Di Falco énumère, concluant que, selon ces cavalieri, il n'y aurait poésie ni politesse en-dehors de ces mots :

montrant à leurs aimées combien elles leur causent de jalousie, disputant de la jalousie, de l'espoir et des autres passions de l'Amour, et que " la Nature, lorsqu'elle vous fit, Madame, ne se préoccupa que de votre beauté ", répétant les étincelles, l'angoisse, les soupirs, les accents cuisants d'Amour, la récompense, la merci, les « Madame, vous êtes un soleil », les " vos yeux sont deux étoiles ». ${ }^{61}$

Di Falco trace ainsi le portrait satirique des pédants pétrarquistes, qui lui reprochent d'avoir ouvert le canon à d'autres poètes - Dante en particulier, peut-on imaginer - , et des tartuffes lubriques qui ne jurent que par le Petrarchino. Il caractérise le pétrarquisme à la fois comme manière littéraire et comme langage mondain. Dans les deux cas, il discrédite les partisans de

60. Benedetto Di Falco, Rimario (Naples : Matthio Canze da Brescia, 1535), fol. Hv vº.

61. Benedetto Di Falco, Rimario (Naples : Matthio Canze da Brescia, 1535), fol. Hv v : « dimostrando a loro amanze quanto siano ingelositi d'elle, disputando di gelosia, dela speranza, e d'altri effetti d'Amore, e che la natura quando fece uoi madonna, fu sol di uostra bellezza sollecita, reitirando lor le fauille, l'angoscia, i sospiri, gl'accenti cocenti d'Amore, il guidardone, la merce, madonna voi sete un sole, gl'occhi sono due stelle». 
la seule imitation de Pétrarque - divin poète, par ailleurs. Reprenant les considérations antiques sur la moralité qui doit être celle de l'homme de lettres, Di Falco accuse ses adversaires de mœurs corrompues. Pour se défendre, il cite à son avantage un célèbre passage de la préface du commentaire d'Isaïe où saint Jérôme opposait les lecteurs des fables milésiennes - symbole de la lascivité de la littérature - à ceux de Platon.

La défense de Di Falco ne manque pas de piquant : "prudentissime », la citation l'est à un double titre, et par ce que Jérôme dit, et par la manière dont Di Falco le cite. En effet, il omet la fin de la pensée de l'auteur de la Vulgate ${ }^{62}$. Celui-ci opposait à Cicéron avouant ses difficultés à comprendre Platon les cohortes d'élèves chahuteurs qui déclament dans les écoles le testament du porcelet "Grunnius Corocotta ». Il s'agissait chez Jérôme d'une référence au Testamentum porcelli, parodie de testament du IV siècle, qui avait fait les délices des lettrés de l'Antiquité tardive ${ }^{63}$. Saint Jérôme conférait ainsi, sans peut-être le vouloir, une renommée à cette petite pièce burlesque, à l'origine d'une riche tradition ${ }^{64}$ qui se prolongea jusque, entre autres, dans le Testamento

62. Saint Jérôme, Libri XII commentariorum in Isaiam, praefatio : " nullus tam imperitus scriptor est qui lectorem non inveniat similem sui, multoque pars maior est Milesias fabellas revolventium quam Platonis libros. in altero enim ludus et oblectatio est, in altero difficultas et sudor mixtus labori. denique Timaeum de mundi harmonia astrorumque cursu et numeris disputantem ipse qui interpretatus est Tullius se non intellegere confitetur, testamentum autem Grunnii Corocottae porcelli decantant in scholis puerorum agmina cachinnantium. » (Euvres de Saint Jérôme, publiées par M. Benoît Matougues, sous la direction de M. L. Aimé-Martin, Paris, Auguste Desrez, 1838, p. 624, trad. légèrement retouchée : «Il n'y a point d'écrivain si sot qui ne trouve un sot lecteur, et le nombre de ceux qui parcourent les fables milésiennes est beaucoup plus considérable que le nombre de ceux qui lisent les livres de Platon ; car, dans l'un, il y a distraction et amusement, dans l'autre, difficulté et travail. Enfin, celui qui a interprété Tullius avance ne pas comprendre Timée, discutant sur l'harmonie du monde, le cours et le nombre des astres. Mais des troupes d'enfants chantent en riant dans les écoles le Testament de Grunhius Corocotta Sorcillus»).

63. Testamentum porcelli : una problematica parodia tardolatina, éd. Bruno Mocci (Innsbruck : s.n., 1981).

64. Transmis au cours du Moyen Âge par de multiples manuscrits, ce Testamentum inspira le Testamentum asini, la Lamentation du lièvre ou encore la Complainte du cygne des Carmina burana. Voir Clarence A. Forbes et Michael S. Ginsburg, "Testamentum Porcelli », Revue de philologie, de littérature et d'histoire anciennes 10 (1936) : 171-181 ; Michel Griffe, "Le Testament du porc et le Testament de l'âne. Deux fables latines, à l'origine d'un genre littéraire ", in Fables et fabulistes. Variations autour de La Fontaine (Paris : Éditions Interuniversitaires, 1992), 125-147; Paolo Poccetti, « Le Testamentum Porcelli, entre philologie et pastiche macaronique ", Revue des études latines 81 (2003) : 252-276 ; Jairo Javier 
dell'Elefante (1516), parfois attribué à l'Arétin ${ }^{65}$. Que Di Falco connût ou non ce Testamento moderne, il est plus que probable que sa citation tronquée de saint Jérôme renvoyait à la tradition à laquelle ce Testamento appartenait et invitait ses lecteurs à identifier les pétrarquistes lascifs avec les lecteurs des fables milésiennes.

Soucieux de parodier, mais sans se trainer dans la boue que supposerait la fin de la citation de saint Jérôme, Di Falco dessine le portrait du pédant pétrarquisant et de l'amant courtisan de manière à les discréditer dans leurs attaques sans lui-même se salir, comme il le précise au début de sa diatribe. Le fin mot de sa satire, déjà virulente, est laissé à l'appréciation de ceux qui, subtils connaisseurs de la parodie humaniste, sauront se souvenir du texte de saint Jérôme et, par-delà celui-ci, du Testamentum porcelli. Les citations tronquées de saint Jérôme servent de canevas à la parodie des pétrarquistes napolitains de Di Falco sans toutefois en expliciter toutes les virtualités.

Franco, en revanche, qui écrirait peu d'années après ses sonnets contre l'Arétin et ses Priapées, ne reculerait pas devant cette limite, qui le ferait passer de la réticente critique napolitaine à un rire autrement plus grossier. Cependant, si les formes que son antipétrarquisme adopta avaient été apprises à Venise, la

García Sánchez, «El Testamentum Porcelli, una fuente de latín vulgar siempre sugerente », in Romanística UM, coord. João Corrêa-Cardoso et Maria Do Céu Fialho (Coimbra : Universidad de Coimbra, 2014), 53-70. Le texte était bien connu au début du XVI e siècle, car il connut non moins de trois éditions en Italie - à Fano, chez les Soncino (Grunii Corococtae Porcelli Testamentum. Laurentii Abstemii Maceratensis Hecatomythium secundum. Eiusdem libellus De verbis communibus [Fano : Gershom ben Moshe Soncino, 1505]), et à Venise, en 1520 et 1524, chez Rusconi -, puis d'autres - ainsi à Bâle, en 1532, chez Christian Wechel (1505). Voir Giacomo Manzoni, Annali tipografici dei Soncino (Bologne : Romagnoli, 1883), vol. III, 15bis, 82. Le plus probable est que Di Falco ait pu connaître cette édition du fait de sa publication avec le petit manuel de grammaire d'Abstemius, le Libellus de verbis communibus. Le livre fut mis à l'Index, aussi bien en Espagne dès 1559, quà Rome, en 1564, et aux Pays-Bas. Voir Jesús Martínez de Bujanda, Index d'Anvers, 1569, 1570, 1571 (Sherbrooke, Paris : Centre d'Études de la Renaissance, Éditions de l'Université de Sherbrooke, Librairie Droz, 1988), 118 et Index de l'Inquisition espagnole 1583, 1584 (Sherbrooke, Paris : Centre d'Études de la Renaissance, Éditions de l'Université de Sherbrooke, Librairie Droz : 1993), 430.

65. Voir Vittorio Rossi, «Un elefante famoso », Intermezzo I (1890) : 625-644, rééd. Scritti di critica letteraria. Dal Rinascimento al Risorgimento (Florence : Sansoni, 1930), 223-242 ; Angelo Romano, «Il 'Testamento dell'elefante' attribuito a Pietro Aretino " [1988], in Periegesi aretiniane. Testi, schede e note biografiche intorno a Pietro Aretino (Rome : Salerno, 1991), 89-108 et Aretino, Teatro, t. I, Cortigiana (1525 e 1534), 44-45. Le Testamento est cité par l'Arétin dans le prologue de la Cortigiana même, comme modèle d'écriture parodique (cf. Aretino, t. I, Cortigiana [1525 e 1534], 66). 
pensée au service de laquelle elles furent placées demeurait napolitaine par ses idéaux poétiques.

\section{Il Petrarchista de Niccolò Franco, un fruit de l'antipétrarquisme napolitain}

En février 1538, Niccolò Franco adressait une lettre burlesque à M. Polo Cicogna. Il s'y demandait pourquoi il n'était pas en son pouvoir d'adresser une requête au Paradis. Et d'imaginer que l'objet en serait d'obtenir la grâce d'« être bon poète et de savoir imiter, autrement dit piller Pétrarque " (" esser buon poeta, e sapere imitare idest rubare il Petrarcha »); à défaut, il demanderait à rejoindre Christophe de Longueil, parmi les Cicéroniens, ou encore Guillaume Budé, parmi les Érasmiens ${ }^{66}$. Pour Franco, les lettres de son temps pâtissaient donc de la servilité de l'imitation. C'est la raison pour laquelle il publia peu après Il Petrarchista, afin de vitupérer non point tant Pétrarque, excellent poète, que les singes et les perroquets qui lui dérobent ses mots et ses images. Les moyens de sa satire - le dialogue à la manière d'Érasme, la parodie burlesque du pétrarquisme, à la façon de Berni et de l'Arétin - étaient d'inspiration vénitienne. En revanche, le fond de cette satire, exprimant une méfiance esthétique envers l'adulation de Pétrarque, venait de sa formation napolitaine.

Les cercles humanistes héritiers de Pontano - inventeur d'une poésie néolatine des plus novatrices - et de Sannazar - auteur de l'Arcadie et du De partu Virginis - avaient en effet manifesté une grande réticence face à l'invasion pétrarquiste lancée par les Prose della volgar lingua. Ces cercles cultivaient tout autant la vis satirica que les milieux humanistes plus septentrionaux : par l'Hermaphrodite, Beccadelli s'était imposé comme le nouveau Martial ; dans le Charon, Pontano s'était fait le pourfendeur de la pédanterie des grammairiens - préfigurant en cela Érasme - ; quant à Sannazar, il s'était rendu célèbre par ses épigrammes contre un Politien réduit à l'état de puce (pulex) et de mouche $(\operatorname{mosca})^{67}$. La satire des faux pétrarquistes du Rimario de

66. Voir Niccolò Franco, Le Pistole Vulgari... (1539), fol. LVIv .

67. Pour l'Hermaphrodite, voir Roland Béhar, «"Fuit obsceni plena tabella ioci” : Antonio Beccadelli et le scandale de l'imitation des Anciens ", in Obscène Moyen Âge ?, éd. Nelly Labère (Paris : Honoré Champion, 2015), 273-314 ; pour Pontano, voir : Giovanni Pontano, Dialogus qui Charon inscribitur, traducción y prólogo de María José Vega, ultílogo de Rosa Navarro (Salamanque : SEMYR, 2004); David Marsh, Lucian and the Latins. Humor and Humanism in the Early Renaissance (Ann Arbor : 
Di Falco, très mesurée et néanmoins animalisante, s’inscrivait dans le droit fil de ces maîtres.

Bien avant d'arriver à Venise en 1536, Niccolò Franco avait donc été à bonne école. En rendant célèbre le personnage qu'il criblait de ses flèches, son Petrarchista ne fit que réunir en une œuvre ambitieuse, encouragée par l'exemple d'Érasme et de l'Arétin, les critiques déjà formulées depuis Naples à l'encontre de cette engeance qui, dès le début des années 1530 - selon le témoignage d'une lettre de Franco lui-même - , portait dans la capitale méridionale le nom de $«$ pétrarquiste $»^{68}$.

University of Michigan Press, 1998), 129-143 ; John Nassichuk, "Sagesse et curiosité dans le Charon de Giovanni Pontano », Camence 15 (2013) : http://www.paris-sorbonne.fr/IMG/pdf/7-Nassichuk.pdf ; pour Sannazar épigrammatiste, Carlo Vecce, « Multiplex hic anguis. Gli epigrammi di Sannazaro contro Poliziano », Rinascimento 30 (1990) : 235-255.

68. On peut citer cette lettre de Franco à Leone Orsini, « da Napoli », du 20 décembre 1531 (date qu'il faut prendre avec précaution, mais qui peut se considérer comme la plus ancienne attestation du terme), Niccolò Franco, Le Pistole Vulgari... (1539), fol. Xr $\mathrm{Xr}^{\circ}$ : Volete conoscere, Signore mio, un poeta? Non guardiate se fa più favore a i vecchi ch'a gli sbarbati, come si dice : perché questo gli è una favola ne la lor poesia [...]. Volete conoscere un pedante che non sa far altro che dar precetti del Dottrinale? Ponete cura se nel raggionare, di secco in secco, mette mano a i Paradossi di Cicerone. Volete conoscere un Petrarchista in vista? Guardiate che non sa fare un sonetto, se non ruba versi o non infilza parole. " ( Monsieur, voulez-vous reconnaître un poète? Ne considérez pas s'il fait plus de faveurs aux vieillards qu'aux imberbes, comme on dit, car ceci n'est qu'une fable de leur poésie [...]. Désirez-vous reconnaître un pédant, qui ne sait rien faire d'autre que de donner des préceptes du Doctrinale ? Regardez si, dans son raisonnement, il se servira tout à fait hors de propos des Paradoxes de Cicéron. Souhaitez-vous reconnaître un Pétrarquiste à la vue ? Observez s'il sait tourner un sonnet sans dérober des vers et enfiler des termes $"$ ). 